\title{
ZONES OF UNIFORM DECOMPOSITION IN TENSOR PRODUCTS ${ }^{1}$
}

\begin{abstract}
ALEX JAY FEINGOLD
ABSTRACT. Let $V_{\lambda}$ be a finite dimensional irreducible module for a complex semisimple Lie algebra. It is shown that the decomposition of tensor products $V_{\lambda} \otimes V_{\tau}$ for all dominant integral weights $\tau$ may be derived from those for a finite set of such $\tau$. An explicit choice of such a finite set (depending on $\lambda$ ) is given.
\end{abstract}

Introduction. Let $L$ be a somplex semisimple Lie algebra with simple roots $\left\{\alpha_{1}, \ldots, \alpha_{l}\right\}$ and fundamental weights $\left\{\omega_{1}, \ldots, \omega_{l}\right\}$. That is, $\left\{\omega_{1}, \ldots, \omega_{l}\right\}$ is a basis of the integral weight lattice, $\Lambda$, such that $\left\langle\omega_{i}, \alpha_{j}\right\rangle=2\left(\omega_{i}, \alpha_{j}\right) /\left(\alpha_{j}, \alpha_{j}\right)$ $=\delta_{i j}$. By definition, $\tau=\sum_{i=1}^{l} m_{i} \omega_{i} \in \Lambda^{+}$if and only if $m_{i} \geqslant 0$ are all integers. Also, $\sum_{i=1}^{l} \omega_{i}=\delta=\frac{1}{2} \sum \alpha$, where $\alpha \in \Phi^{+}$is the set of all positive roots. All $L$-modules in this paper are finite dimensional.

Let $W$ denote the Weyl group of $L . W$ is generated by the simple reflections $\left\{\sigma_{1}, \ldots, \sigma_{l}\right\}$, where $\sigma_{i}(x)=x-\left\langle x, \alpha_{i}\right\rangle \alpha_{i}$. For any $i, 1 \leqslant i \leqslant l$, we define $W(i)$ to be the subgroup of $W$ generated by $\left\{\sigma_{j} \mid j \neq i, 1 \leqslant j \leqslant l\right\}$. Note that each element of $W(i)$ fixes $\omega_{i}$.

In all of what follows, the set of weights of the irreducible $L$-module $V_{\lambda}$ will be denoted by $\Pi$.

We shall prove

THEOREM 1. Let $V_{\lambda}$ be the irreducible L-module of highest weight $\lambda$. Let $\tau=\sum_{j=1}^{l} m_{j} \omega_{j} \in \Lambda^{+}$and $V_{\lambda} \otimes V_{\tau}=\sum_{\gamma \in \Lambda^{+}} r_{\gamma} V_{\gamma}$. Then for each $i, 1 \leqslant i \leqslant l$, there is a positive integer $n_{i}$, depending only on $\lambda$, such that if $m_{i} \geqslant n_{i}$, then $V_{\lambda} \otimes V_{\tau+\omega_{i}}=\Sigma_{\gamma \in \Lambda^{+}} r_{\gamma} V_{\gamma+\omega_{i}}$.

We shall give explicit values for the $n_{i}$ in terms of $\lambda$.

Theorem 1 should be compared with a result of Kostant [4]. He puts a much stronger requirement on $\tau$, namely that $\mu+\tau$ is dominant for every $\mu \in \Pi$. Under this condition, one can read off the decomposition of $V_{\lambda} \otimes V_{\tau}$ from the weight-space decomposition of $V_{\lambda}: V_{\lambda} \otimes V_{\tau}=\Sigma_{\mu \in \Pi} \operatorname{Mult}_{\lambda}(\mu) V_{\mu+\tau}$. The conclusion of Theorem 1 clearly follows for such $\tau$. However, Kostant's condition is satisfied only by dominant weights $\tau$ well into the interior of the fundamental chamber, and gives no information about infinitely many weights on or near the chamber walls. Theorem 1, on the other hand,

Received by the editors June 2, 1976 and, in revised form, September 20, 1976.

AMS (MOS) subject classifications (1970). Primary 17B10; Secondary 81A78.

${ }^{1}$ Research partially supported by NSF research grant MPS72-04643. 
expresses a condition of uniformity along lines in the decomposition of the tensor product $V_{\lambda} \otimes V_{\tau}$ whenever $\tau$ is outside a specified finite region.

If we let $S(i)=\cup_{\sigma \in W(i)} \sigma\left(\Lambda^{+}\right)$, then $n_{i}$ may be chosen as the least positive integer such that for each $\mu \in \Pi$ we have $\mu+n_{i} \omega_{i} \in S(i)$.

Corollary 1. Let $V_{\lambda}$ be fixed. Let $\left(n_{1}, \ldots, n_{l}\right)$ be the l-tuple of positive integers which can be found by the above theorem. If we know the decompositions into irreducible $L$-modules of the finite set of tensor products $\left\{V_{\lambda} \otimes\right.$ $V_{\tau} \mid \tau=\sum_{j=1}^{l} m_{j} \omega_{j}$ and $m_{j} \leqslant n_{j}$ for all $\left.j, 1 \leqslant j \leqslant l\right\}$, then we know the decomposition of the tensor product of $V_{\lambda}$ with any irreducible L-module.

Let $i, 1 \leqslant i \leqslant l$, be fixed throughout the following and let $S=S(i)$.

LEMMA 1. $\cup_{\sigma \in W(i)} \sigma\left(\Lambda^{+}\right)=\left\{x \in \Lambda \mid\left(x, \sigma \alpha_{i}\right) \geqslant 0, \forall \sigma \in W(i)\right\}$.

Proof. Let $S=\cup_{\sigma \in W(i)} \sigma\left(\Lambda^{+}\right)$and $S^{\prime}=\left\{x \in \Lambda \mid\left(x, \sigma \alpha_{i}\right) \geqslant 0, \forall \sigma \in\right.$ $W(i)\}$. If $x \in \Lambda^{+}$then $\left(x, \alpha_{j}\right) \geqslant 0$ for $1 \leqslant j \leqslant l$, so for any $\alpha \in \Phi^{+},(x, \alpha)$ $>0$. For any $\sigma \in W(i), \sigma \alpha_{i} \in \Phi^{+}$because it is certainly a root and has +1 as its $\alpha_{i}$ coefficient, so all coefficients are nonnegative. It follows that $\left(x, \sigma \alpha_{i}\right) \geqslant 0$; that is, $x \in S^{\prime}$. Thus, $\Lambda^{+} \subseteq S^{\prime}$. For any $x \in S^{\prime}$ and any $\sigma$, $\sigma^{\prime} \in W(i),\left(\sigma x, \sigma^{\prime} \alpha_{i}\right)=\left(x, \sigma^{-1} \sigma^{\prime} \alpha_{i}\right) \geqslant 0$ since $\sigma^{-1} \sigma^{\prime} \in W(i)$. This means that if $x \in S^{\prime}$ then $\sigma x \in S^{\prime}$ for any $\sigma \in W(i)$. From $\Lambda^{+} \subseteq S^{\prime}$ we then get $S \subseteq S^{\prime}$.

Suppose there is an $x \in S^{\prime}, x \notin S$. In the finite set $\{\sigma x \mid \sigma \in W(i)\}$ let $\sigma x$ be chosen such that $(\sigma x, \delta)$ is maximal. Since $x \notin S, \sigma x \notin \Lambda^{+}$and there is a $j, 1 \leqslant j \leqslant l$, such that $\left(\sigma x, \alpha_{j}\right)<0$. If $j \neq i$ then $\sigma_{j} \in W(i)$ and $\sigma_{j} \sigma \in W(i)$. But $\left(\sigma_{j} \sigma x, \delta\right)=\left(\sigma x, \sigma_{j} \delta\right)=\left(\sigma x, \delta-\alpha_{j}\right)=(\sigma x, \delta)-\left(\sigma x, \alpha_{j}\right)>(\sigma x, \delta)$, contradicting the choice of $\sigma x$. So $j=i$ and $\left(x, \sigma^{-1} \alpha_{i}\right)=\left(\sigma x, \alpha_{i}\right)<0$. But since $\sigma^{-1} \in W(i)$, this contradicts $x \in S^{\prime}$, giving $S=S^{\prime}$.

LEMMA 2. There is an integer $n_{i} \geqslant 0$ such that for any $\mu \in \Pi, \mu+n_{i} \omega_{i} \in S$. The least such $n_{i}$ is $\operatorname{Max}\left\{\left\langle\mu, \alpha_{\mathrm{i}}\right\rangle \mid \mu \in \Pi\right\}$.

Proof. For any $\sigma \in W(i),\left(n_{i} \omega_{i}, \sigma \alpha_{i}\right)=n_{i}\left(\sigma^{-1} \omega_{i}, \alpha_{i}\right)=n_{i}\left(\omega_{i}, \alpha_{i}\right)=$ $n_{i}\left(\alpha_{i}, \alpha_{i}\right) / 2$. The conditions on $n_{i}$ equivalent to $\mu+n_{i} \omega_{i} \in S$ for all $\mu \in \Pi$ are $0 \leqslant\left(\mu+n_{i} \omega_{i}, \sigma \alpha_{i}\right)=\left(\mu, \sigma \alpha_{i}\right)+\left(n_{i} \omega_{i}, \sigma \alpha_{i}\right)=\left(\mu, \sigma \alpha_{i}\right)+n_{i}\left(\alpha_{i}, \alpha_{i}\right) / 2$ for all $\mu$ $\in \Pi$ and all $\sigma \in W(i)$. That is, $n_{i} \geqslant-2\left(\mu, \sigma \alpha_{i}\right) /\left(\alpha_{i}, \alpha_{i}\right)=-\left\langle\mu, \sigma \alpha_{i}\right\rangle=$ $-\left\langle\sigma^{-1} \mu, \alpha_{i}\right\rangle=\left\langle\sigma^{-1} \mu, \sigma_{i} \alpha_{i}\right\rangle=\left\langle\sigma_{i} \sigma^{-1} \mu, \alpha_{i}\right\rangle$. Since $\Pi$ is invariant under $W$, $\left\{\sigma_{i} \sigma^{-1} \mu \mid \mu \in \Pi, \sigma \in W(i)\right\}=\Pi$. We now have the finite number of conditions $n_{i} \geqslant\left\langle\mu, \alpha_{i}\right\rangle$ for all $\mu \in \Pi$ which has least solution

$$
n_{i}=\operatorname{Max}\left\{\left\langle\mu, \alpha_{i}\right\rangle \mid \mu \in \Pi\right\} \geqslant 0 \text {. }
$$

Lemma 3. For any $\gamma_{1}, \gamma_{2} \in S, \gamma_{1}+\gamma_{2} \in S$.

Proof. Clear from Lemma 1.

Proof of THEOREM 1. If we use the notation

$$
T_{\lambda}=\sum_{\sigma \in W} \operatorname{sgn}(\sigma) \exp (\sigma(\lambda+\delta)),
$$


then the Weyl character formula says $X_{\lambda} \cdot T_{0}=T_{\lambda}$, where $X_{\lambda}$ is the character of a representation $V_{\lambda}$ of highest weight $\lambda$. Then the character of $V_{\lambda} \otimes V_{\tau}$ is $X_{\lambda} \cdot X_{\tau}$. After some elementary manipulations, one sees that

$$
X_{\lambda} \cdot X_{\tau} \cdot T_{0}=\sum_{\mu \in \Pi} \operatorname{Mult}_{\lambda}(\mu) \cdot T_{\mu+\tau} \cdot
$$

Replacing $\tau$ by $\tau+\omega_{i}$, we also have

$$
X_{\lambda} \cdot X_{\tau+\omega_{i}} \cdot T_{0}=\sum_{\mu \in \Pi} \operatorname{Mult}_{\lambda}(\mu) \cdot T_{\mu+\tau+\omega_{i}}
$$

By Lemma $2, \mu+n_{i} \omega_{i} \in S$ for all $\mu \in \Pi$. If $\tau=\sum_{j=1}^{l} m_{j} \omega_{j}$ satisfies $m_{i}>n_{i}$, then $\tau-n_{i} \omega_{i} \in \Lambda^{+} \subseteq S$. By Lemma 3, $\mu+\tau=\left(\mu+n_{i} \omega_{i}\right)+(\tau-$ $\left.n_{i} \omega_{i}\right) \in S$. Of course, $\omega_{i}, \delta \in \Lambda^{+} \subseteq S$ and so $\mu+\delta+\tau \in S$ as well as $\mu+\delta+\tau+\omega_{i} \in S$. This means that both $\mu+\delta+\tau$ and $\mu+\delta+\tau+\omega_{i}$ are conjugate by elements of $W(i)$ to dominant weights. In fact, they are conjugate by the same element because if $\sigma_{\mu}(\mu+\delta+\tau) \in \Lambda^{+}$for $\sigma_{\mu} \in$ $W(i)$ then $\sigma_{\mu}\left(\mu+\delta+\tau+\omega_{i}\right)=\sigma_{\mu}(\mu+\delta+\tau)+\omega_{i} \in \Lambda^{+}$. Thus

$$
\begin{aligned}
T_{\mu+\tau} & =\sum_{\sigma \in W} \operatorname{sgn}(\sigma) \exp (\sigma(\mu+\tau+\delta)) \\
& =\sum_{\sigma \in W} \operatorname{sgn}(\sigma) \operatorname{sgn}\left(\sigma_{\mu}\right) \exp \left(\sigma\left(\sigma_{\mu}(\mu+\tau+\delta)\right)\right)
\end{aligned}
$$

and

$$
\begin{aligned}
T_{\mu+\tau+\omega_{i}} & =\sum_{\sigma \in W} \operatorname{sgn}(\sigma) \exp \left(\sigma\left(\mu+\tau+\omega_{\mathrm{i}}+\delta\right)\right) \\
& =\sum_{\sigma \in W} \operatorname{sgn}(\sigma) \operatorname{sgn}\left(\sigma_{\mu}\right) \exp \left(\sigma\left(\sigma_{\mu}(\mu+\tau+\delta)+\omega_{\mathrm{i}}\right)\right)
\end{aligned}
$$

This means that $T_{\mu+\tau} / T_{0}=\operatorname{sgn}\left(\sigma_{\mu}\right) \cdot X_{\sigma_{\mu}(\mu+\tau+\delta)-\delta}$ and $T_{\mu+\tau+\omega_{i}} / T_{0}=\operatorname{sgn}\left(\sigma_{\mu}\right)$ $\cdot X_{\sigma_{\mu}(\mu+\delta+\tau)-\delta+\omega_{i}}$. Then

$$
X_{\lambda} \cdot X_{\tau}=\sum_{\mu \in \Pi} \operatorname{Mult}_{\lambda}(\mu) \cdot \operatorname{sgn}\left(\sigma_{\mu}\right) \cdot X_{\sigma_{\mu}}(\mu+\tau+\delta)-\delta
$$

and

$$
X_{\lambda} \cdot X_{\tau+\omega_{i}}=\sum_{\mu \in \Pi} \operatorname{Mult}_{\lambda}(\mu) \cdot \operatorname{sgn}\left(\sigma_{\mu}\right) \cdot X_{\sigma_{\mu}(\mu+\tau+\delta)-\delta+\omega_{i}}
$$

Grouping equivalent terms together, if $X_{\lambda} \cdot X_{\tau}=\Sigma_{\gamma \in \Lambda^{+}} r_{\gamma} X_{\gamma}$ then the above shows that $X_{\lambda} \cdot X_{\tau+\omega_{i}}=\Sigma_{\gamma \in \Lambda^{+}} r_{\gamma} X_{\gamma+\omega_{i}}$ as claimed by the theorem.

It should be noted that the author originally based his proof on a formula of Klimyk [3], whose geometric nature was essential to the discovery of this result. The present proof, based on the closely related and well-known Weyl character formula, was suggested by the referee.

Note that if $\tau=\sum_{j=1}^{l} m_{j} \omega_{j}$ satisfies $m_{j} \geqslant n_{j}$ for all $j, 1 \leqslant j \leqslant l$, then by Lemma 2, for each $\mu \in \Pi$ and each $j, \mu+n_{j} \omega_{j} \in S(j)$ and $\tau-n_{j} \omega_{j} \in \Lambda^{+} \subseteq$ $S(j)$. Then $\mu+\tau \in S(j)$, which means $\mu+\tau \in \cap_{1<j<l} S(j)$ for all $\mu \in \Pi$.

For each $j, \Lambda^{+} \subseteq S(j)$, so $\Lambda^{+} \subseteq \cap_{1 \leqslant j \leqslant l} S(j)$. For each $j, S(j) \subseteq\{x \in$ 
$\left.\Lambda \mid\left(x, \alpha_{j}\right) \geqslant 0\right\}$. This means that $\cap_{1 \leqslant j \leqslant l} S(j) \subseteq\left\{x \in \Lambda \mid\left(x, \alpha_{j}\right) \geqslant 0\right.$ for all $1 \leqslant j \leqslant l\}=\Lambda^{+}$. We now have $\bigcap_{1 \leqslant j \leqslant l} S(j)=\Lambda^{+}$.

The above says that for each $\mu \in \Pi, \mu+\tau \in \Lambda^{+}$, which is the condition required by Kostant's theorem. Although Kostant used a theorem of Brauer [1] in his proof, Weyl's formula also gives the result as follows. If $\mu+\tau \in \Lambda^{+}$ then $\mu+\delta+\tau$ is strictly dominant, so $\sigma_{\mu}=1$ and $X_{\sigma_{\mu}(\mu+\delta+\tau)-\delta}=X_{\mu+\tau}$. Weyl's formula then says $X_{\lambda} \cdot X_{\tau}=\Sigma_{\mu \in \Pi} \operatorname{Mult}_{\lambda}(\mu) \cdot X_{\mu+\tau}$ which is now a direct sum.

In fact, if $\tau_{0}=\sum_{j=1}^{l} n_{j} \omega_{j}$ then no "smaller" dominant weight $\tau^{\prime}$ satisfies $\mu+\tau^{\prime} \in \Lambda^{+}$for all $\mu \in \Pi$. If we let $\tau^{\prime}=\sum_{j=1}^{l} q_{j} \omega_{j}$ with some $q_{p}<n_{p}$, then the condition $\mu+\tau^{\prime} \in \Lambda^{+}$for all $\mu \in \Pi$ means $\left\langle\mu+\tau^{\prime}, \alpha_{i}\right\rangle \geqslant 0$ for $1 \leqslant i \leqslant$ l. That is, $\left\langle\mu, \alpha_{i}\right\rangle+\sum_{j=1}^{l} q_{j}\left\langle\omega_{j}, \alpha_{i}\right\rangle=\left\langle\mu, \alpha_{i}\right\rangle+q_{i} \geqslant 0$. Or $q_{i} \geqslant-\left\langle\mu, \alpha_{i}\right\rangle=$ $\left\langle\mu, \sigma_{i} \alpha_{i}\right\rangle=\left\langle\sigma_{i} \mu, \alpha_{i}\right\rangle$ for all $\mu \in \Pi$ and $1 \leqslant i \leqslant l$. As in Lemma $2,\left\{\sigma_{i} \mu \mid \mu \in\right.$ $\Pi\}=\Pi$, so $q_{i} \geqslant\left\langle\mu, \alpha_{i}\right\rangle$. But from Lemma $2, n_{p}=\operatorname{Max}\left\{\left\langle\mu, \alpha_{p}\right\rangle \mid \mu \in \Pi\right\}$. Therefore $q_{p}>n_{p}$, which contradicts $q_{p}<n_{p}$. This shows that the Kostant region of uniform decomposition is precisely $\left\{\tau_{0}+\gamma \mid \gamma \in \Lambda^{+}\right\}$.

LeMMA 4. $n_{i}=\operatorname{Max}\left\{\left\langle\mu, \sigma \alpha_{i}\right\rangle \mid \mu \in \Pi \cap \Lambda^{+}, \sigma \in W\right\}$.

Proof. From Lemma 2, $n_{i}=\operatorname{Max}\left\{\left\langle\mu, \alpha_{i}\right\rangle \mid \mu \in \Pi\right\}$. Every $\mu \in \Pi$ is conjugate to some dominant weight in $\Pi$, giving the lemma.

We can now give the sharper result.

LEMMA 5. $n_{i}=\left\langle\lambda, \theta_{i} \alpha_{i}\right\rangle$ where $\theta_{i} \in W$ is such that $\theta_{i} \alpha_{i}$ is the highest root conjugate to $\alpha_{i}$. Thus, for $L$ simple, if $\alpha_{i}$ is a short root, $\theta_{i} \alpha_{i}$ is the highest short root, and if $\alpha_{i}$ is a long root, $\theta_{i} \alpha_{i}$ is the highest long root.

Proof. Fix $\mu \in \Pi \cap \Lambda^{+}$. Then for any $\sigma \in W,\left\langle\mu, \sigma \alpha_{i}\right\rangle=$ $2\left(\mu, \sigma \alpha_{i}\right) /\left(\alpha_{i}, a_{i}\right)$ and $\left\langle\mu, \theta_{i} \alpha_{i}\right\rangle-\left\langle\mu, \sigma \alpha_{i}\right\rangle=2\left(\mu, \theta_{i} \alpha_{i}-\sigma \alpha_{i}\right) /\left(\alpha_{i}, \alpha_{i}\right) \geqslant 0$ since $\mu$ is dominant and $\theta_{i} \alpha_{i}-\sigma \alpha_{i}$ is a nonnegative sum of positive roots. So $n_{i}=\operatorname{Max}\left\{\left\langle\mu, \theta_{i} \alpha_{i}\right\rangle \mid \mu \in \Pi \cap \Lambda^{+}\right\}$. It is a well-known fact that $\theta_{i} \alpha_{i}$ is dominant, and since $\lambda-\mu$ is a nonnegative sum of positive roots, we have $\left\langle\lambda, \theta_{i} \alpha_{i}\right\rangle-\left\langle\mu, \theta_{i} \alpha_{i}\right\rangle=\left\langle\lambda-\mu, \theta_{i} \alpha_{i}\right\rangle \geqslant 0$. This says the maximum is attained at $\left\langle\lambda, \theta_{i} \alpha_{i}\right\rangle$.

This precise characterization of $n_{i}$ allows us to calculate the $l$-tuple, $\left(n_{1}, \ldots, n_{l}\right)$, for each type of algebra in terms of $\lambda=\sum_{i=1}^{l} m_{i} \omega_{i}$. I have labeled the Dynkin diagrams as in [2]. The results are:

$$
\begin{aligned}
A_{l}: n_{i} & =m_{1}+m_{2}+\cdots+m_{l} \text { for } 1 \leqslant i \leqslant l, \\
B_{l}: n_{i} & =m_{1}+2 m_{2}+\cdots+2 m_{l-1}+m_{l} \text { for } 1 \leqslant i \leqslant l-1, \\
n_{l} & =2 m_{1}+2 m_{2}+\cdots+2 m_{l-1}+m_{l}, \\
C_{l}: n_{i} & =m_{1}+2 m_{2}+\cdots+2 m_{l-1}+2 m_{l} \text { for } 1 \leqslant i \leqslant l-1, \\
n_{l} & =m_{1}+m_{2}+\cdots+m_{l-1}+m_{l}, \\
D_{l}: n_{i} & =m_{1}+2 m_{2}+\cdots+2 m_{l-2}+m_{l-1}+m_{l} \text { for } 1 \leqslant i \leqslant l, \\
E_{6}: n_{i} & =m_{1}+2 m_{2}+2 m_{3}+3 m_{4}+2 m_{5}+m_{6} \text { for } 1 \leqslant i \leqslant 6, \\
E_{7}: n_{i} & =2 m_{1}+2 m_{2}+3 m_{3}+4 m_{4}+3 m_{5}+2 m_{6}+m_{7} \text { for } 1 \leqslant i \leqslant 7,
\end{aligned}
$$




$$
\begin{aligned}
E_{8}: n_{i} & =2 m_{1}+3 m_{2}+4 m_{3}+6 m_{4}+5 m_{5}+4 m_{6}+3 m_{7}+2 m_{8} \\
F_{4}: & \text { for } 1 \leqslant i \leqslant 8, \\
n_{1} & =n_{2}=2 m_{1}+3 m_{2}+2 m_{3}+m_{4}, \\
n_{3} & =n_{4}=2 m_{1}+4 m_{2}+3 m_{3}+2 m_{4}, \\
G_{2}: n_{1} & =2 m_{1}+3 m_{2}, \\
n_{2} & =m_{1}+2 m_{2} .
\end{aligned}
$$

For $L$ semisimple, these formulas are applied to each simple component separately. If $\alpha_{i}$ is in a certain component of the Dynkin diagram of $L$, then the highest root conjugate to $\alpha_{i}$ involves only the roots in that component. So $n_{i}$ is calculated according to the type of that component and is given by one of the above formulas involving only those $m_{j}$ such that $\alpha_{j}$ is in that component.

\section{REFERENCES}

1. R. Brauer, Sur la multiplication des caractéristiques des groupes continus et semi-simples, C. R. Acad. Sci. Paris Sér A-B. 204 (1937), 1784-1786.

2. J. E. Humphreys, Introduction to Lie algebras and representation theory, Graduate Texts in Math., no. 9, Springer-Verlag, New York, 1972.

3. A. U. Klimyk, Decomposition of a tensor product of irreducible representations of a semisimple Lie algebra into a direct sum of irreducible representations, Ukrain. Mat. Z. 18 (1966), 19-27; English transl., Amer. Math. Soc. Transl. (2) 76 (1968), 63-73.

4. B. Kostant, A formula for the multiplicity of a weight, Trans. Amer. Math. Soc. 93 (1959), 53-73.

Department of Mathematics, Yale University, Box 2155, Yale Station, New Haven, CONNECTICUT 06520

Current address: Department of Mathematics, Drexel University, Philadelphia, Pennsylvania 19104 